\title{
Assisting better decision-making of geotechnical slope design by using in-house software at BHP Iron Ore
}

\author{
A Maldonado BHP, Australia \\ A Haile BHP, Australia \\ C Meegamarachchi BHP, Australia \\ L Sasmita BHP, Australia
}

\begin{abstract}
This paper presents the Geotechnical Workbench software that was developed by a working team in BHP Iron Ore with the intention of providing an integrated tool to the geotechnical team that permits consistent decision-making of strength properties for slope design. This paper includes a description of the steps taken for software development and the list of learnings gained by the working team. This information is shared for the wider industry for the benefit of others that may endeavour a similar initiative. The software architecture is presented in a very minimalistic manner to protect intellectual property, however, the paper still shows the general idea of software functionalities including some description for the major tools developed within the whole package.
\end{abstract}

Keywords: software, decision-making, slope design

\section{Introduction}

A key component of mining exploration studies is the collection of geotechnical data through diamond core drilling, geophysical tools and subsequent laboratory testing of diamond core samples, with the main objective of providing sufficient data quantity of adequate quality to ensure a reliable assessment of strength properties later used for slope design of future open cuts. Thus, efficient consumption of the data collection has been a major concern of the geotechnical team.

The major motivation for creating an internal tool for geotechnical data analysis originates from the need to standardise geotechnical design process within Pilbara Iron Ore, as envisaged in early 2015 by the overall strategy of the leadership team of planning and technical in BHP Iron Ore. The software would enable standard tools to support engineers with better decision-making and increased efficiency by minimising work-related to repetitive tasks, and hence redirecting their efforts towards slope design optimisation (Snodgrass \& Cockerill 2017).

The author was appointed to create the architecture of the software whilst two developers, who are proficient in the use of Microsoft DotNet technology and C\# language, were assigned to create the software. The process was concluded successfully after three years of intermittent development.

The paper describes the development of this in-house, fit-for-purpose software including a brief explanation of design principles used for slope design in BHP Iron Ore, overall review of the architecture of Geotechnical Workbench, description of some of the major tools created for enabling decision-making and concludes with learnings taken across the two teams: the technology group and geotechnical members.

\section{$2 \quad$ Slope design principles within the software development}

The fundamental philosophy adopted for slope design in BHP Iron Ore obeyed some general ideas:

- Geotechnical analysis should be unbiased and factual. 
- Geotechnical data analysis should account for spatial variability and geological setting to define domains.

- Geotechnical characterisation should aim for the best representation of ground conditions and account for sample size to estimate the degree of confidence of derived parameters.

- The development of in-house software has considered the philosophy above, guiding the users to examine facts, analyse bias, minimise errors, understand sample size and the effect of variability of inputs into data outputs.

- From the geotechnical point of view, Workbench software has incorporated the following assumptions in the construction of the code:

1. Rock mass classification systems adopted for empirical estimation of rock mass strength properties including both the RMR89 (Bieniawski 1989) and the geological strength index (GSI) model classification systems (Marinos et al. 2007; Hoek et.al. 2013). Other models like Q-system (Barton et.al. 1974) were not included as estimates of shear strength models for rock masses are derived using the Hoek \& Brown empirical failure criterion.

2. The intact rock strength is based on the calibration of field estimate strength (FES) recorded along the diamond core data sets against valid uniaxial compressive strength (UCS) test results.

3. Rock quality designation (RQD) is recorded during diamond core logging and is adjusted from photograph records if required.

4. Defect conditions are recorded using RMR89 discontinuity classifications.

5. Defect shear strength is determined using laboratory testing (Goodman 1989; Maldonado 2013; Maldonado \& Mercer 2015) and waviness angle derived from televiewer imaging.

6. Soil shear strength is defined from soil triaxial testing using linear regression best fit and soil classification to sub-group data sets within similar soil horizons (Lancellota 2009).

\section{The architecture of Geotechnical Workbench}

The software architecture is intended to facilitate the state-of-the-art in the derivation of strength properties for slope design, as applied by geotechnical engineers in mining consultancy and other iron ore mining companies. However, the conceptual design stage was complicated by the fact that geotechnical practitioners tended to use different approaches. Hence, the generation of a unique process flow was required to facilitate a fit-for-purpose approach (i.e. standardisation of process).

The process flow used in Geotechnical Workbench was initially developed by gathering all available MS Excel spreadsheets used for data analysis and interpretation of strength properties. Spreadsheets were then simplified by merging and standardising the methods of analyses (Figure 1). 


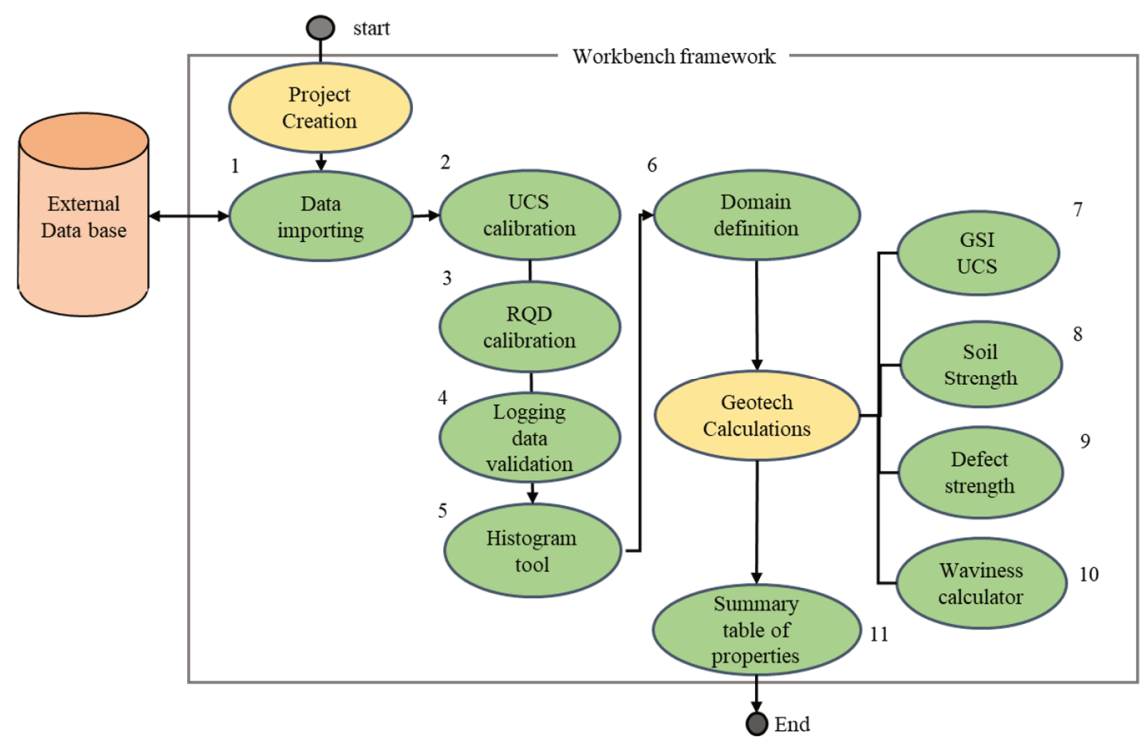

Figure 1 Simplified architecture developed for Geotechnical Workbench. The rectangle represents the framework for the software. Halos represent tools developed within the software, whilst the numbers next to each 'halo' represent the major tools developed for data processing and analysis. The numbers are used here for further description within the text below

Figure 1 presents the architecture of the software, which includes 11 major modules, each of which is briefly described below:

1. Upon project creation, data is imported from an external database using a data importing tool. This permits the connection to the existing database.

2. UCS calibration tool (Figure 2) is used to adjust the logged field estimated strength (FES) by comparison with valid UCS laboratory testing, whilst identification of human bias is possible by including filters to demonstrate possible overestimation or underestimation of the intact rock strength.

3. RQD calibration tool (Figure 3): The RQD , as measured during core logging, is compared to core photographs to verify that RQD\% is acceptable and does not contradict expectations from previous observations. Figure 3 presents a screenshot of the tool with explanatory notes on how the actual tool works.

4. Logging data validation tool (Figure 4): Data is compared to ensure consistency, allowing for standard Excel spreadsheet functionalities for data cleansing, copying and pasting of data sets. Calibration of diamond core data is carried out based on simple rules of consistency (e.g. rock strength intervals should contain some description about structural readings).

5. Histogram tool (Figure 5): Data visualisation with histograms for raw data (point or interval data). Figure 6 presents a screenshot of the tool with explanatory notes on how the actual tool works.

6. Domain definition tool: This module allows data grouping by stratigraphy, weathering, lithology, strength or any other parameter.

7. GSI calculation tool (Figure 6): Statistical tool to present the GSI per domain with statistics, whilst permitting some filters for rock strength. Includes tools for rapid visualisation of standard histograms, calculation of length-weighted median, length-weighted averages and lower bound values.

8. Soil strength calculation tool (Figure 7): Linear best fit-regression analyses with percentiles are used to estimate effective shear strength properties of soil materials from consolidated undrained triaxial testing. 
9. Defect shear strength: Standard non-linear best fit analysis is used to define peak shear strength values for defects (Hencher 2012; Maldonado 2013).

10.This software allows for the calculation of waviness angles per rock units using structural picks, which are interpreted from televiewer images in separate software.

11.Design summary table (Figure 8): This is a decision-making tool, which allows for final selection of central estimates (typically mode or median) and lower bound estimates of rock properties (typically, the first quartile or mean minus 1 standard deviation).

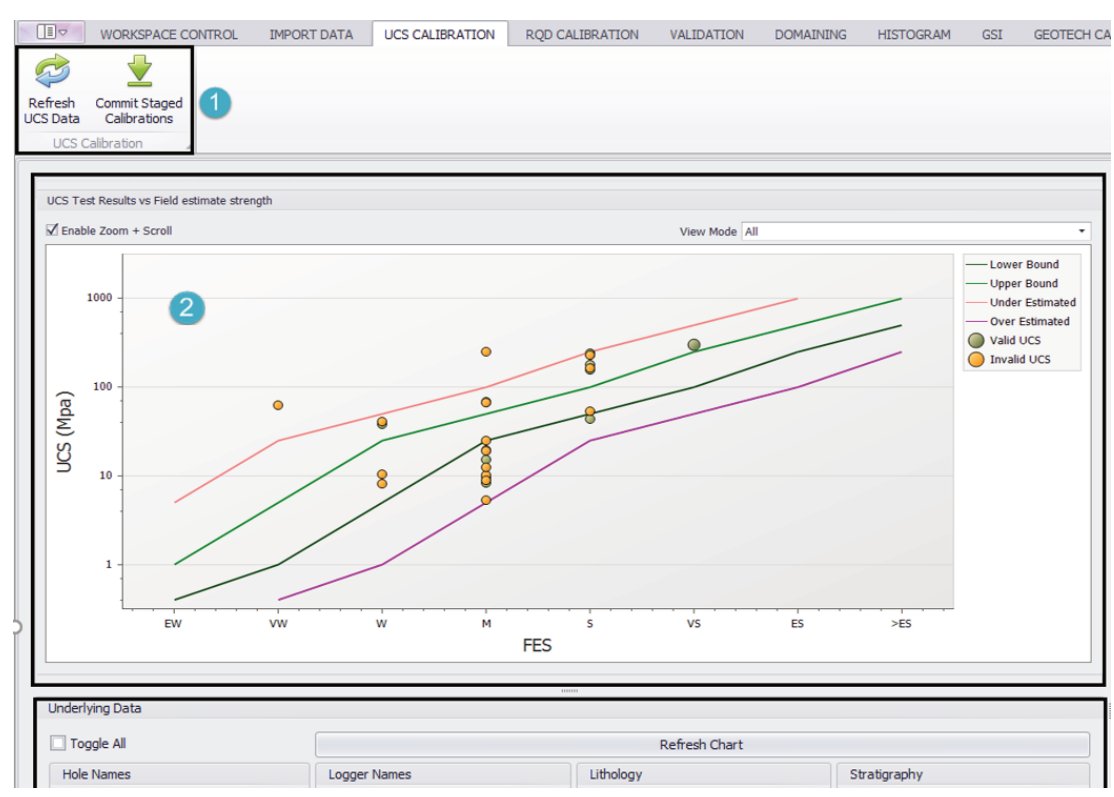

Figure 2 Rock strength calibration using uniaxial compressive strength (UCS) valid testing to adjust the field estimated strength (FES) which is used for approximation of the intact rock strength (i.e. assumed to represent the overall population of UCS for later analysis). Panel 1 activates data display and saves changes. Panel 2 presents the data as a scatter plot for calibration of FES
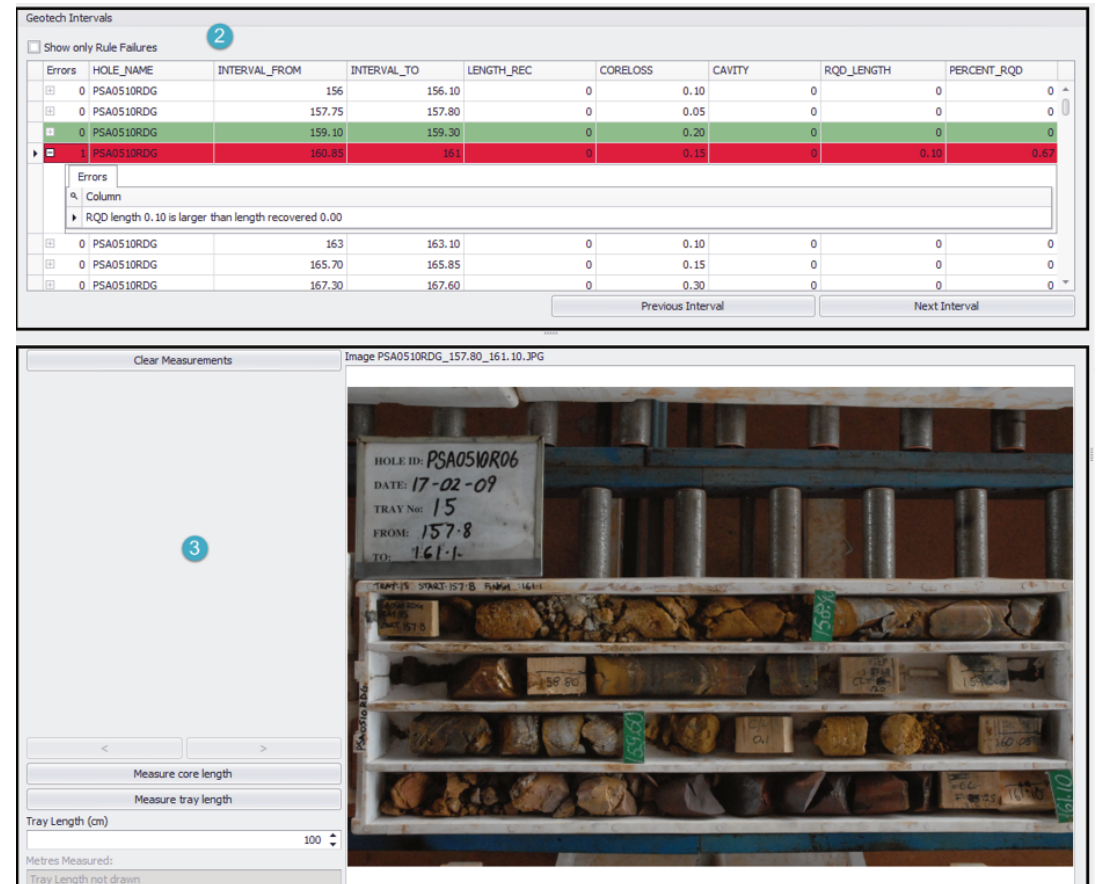

Figure 3 Calibration of logged rock quality designation (RQD) using diamond core photographs to visually verify the length of broken cores and those larger than $10 \mathrm{~cm}$ 
Figure 4 presents the review of the assumptions made by loggers during diamond core logging. This tool permits to set a series of 'customised' validation rules, which are run to detect possible errors recorded during data collection. Note that these rules are for validation only and need manual alterations to change the data (i.e. no auto-fix). As an example, it would not be impossible to get RQD $=100$ for $1+$ sets if they are widely spaced (e.g. three bedding breaks and one joint).

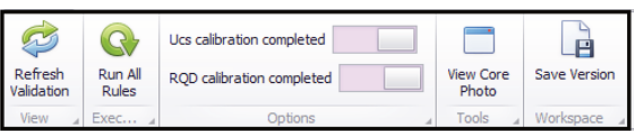

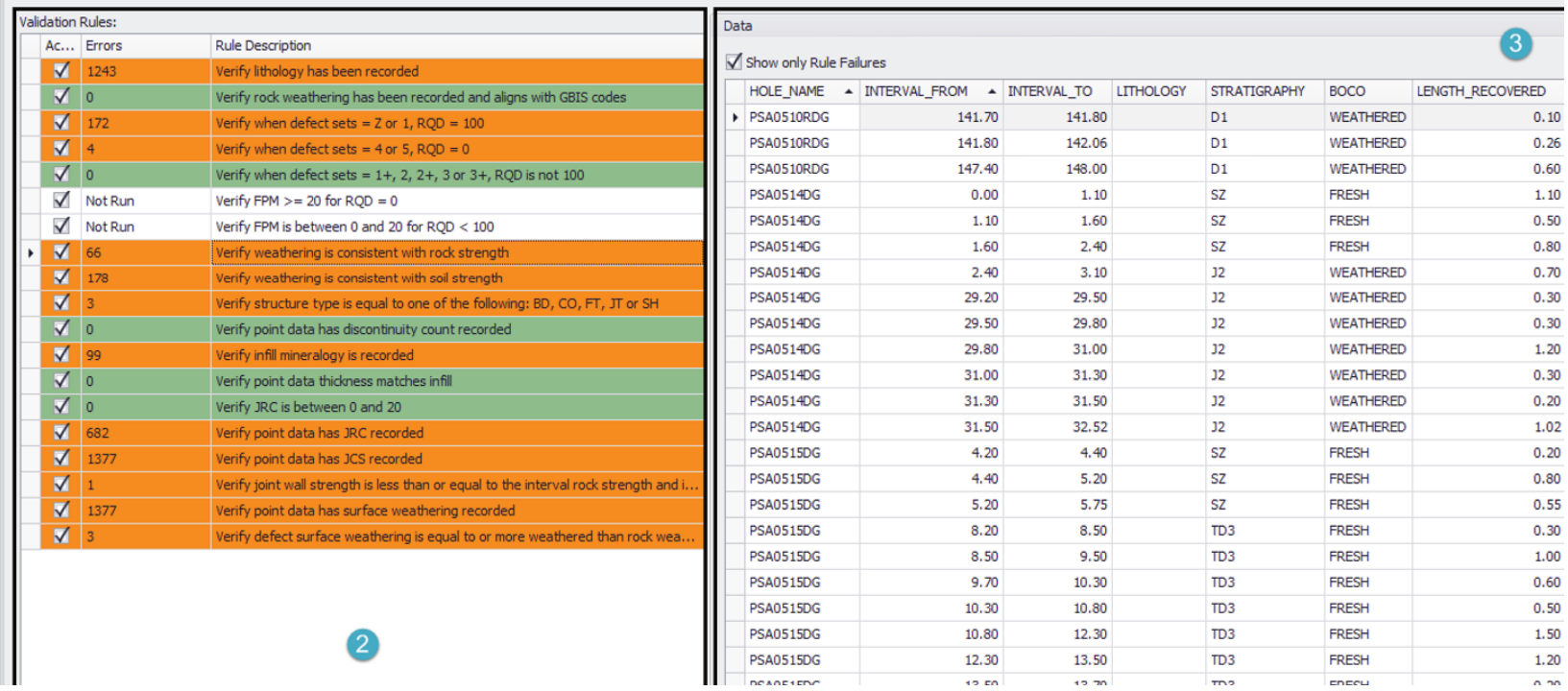

Figure 4 This is the validation module which allows for detection of inconsistences during data collection. (1) This panel represents the execution tool; (2) In green and orange colour to indicate 'correct' and 'inconsistent' assumptions; (3) Actual data to modify

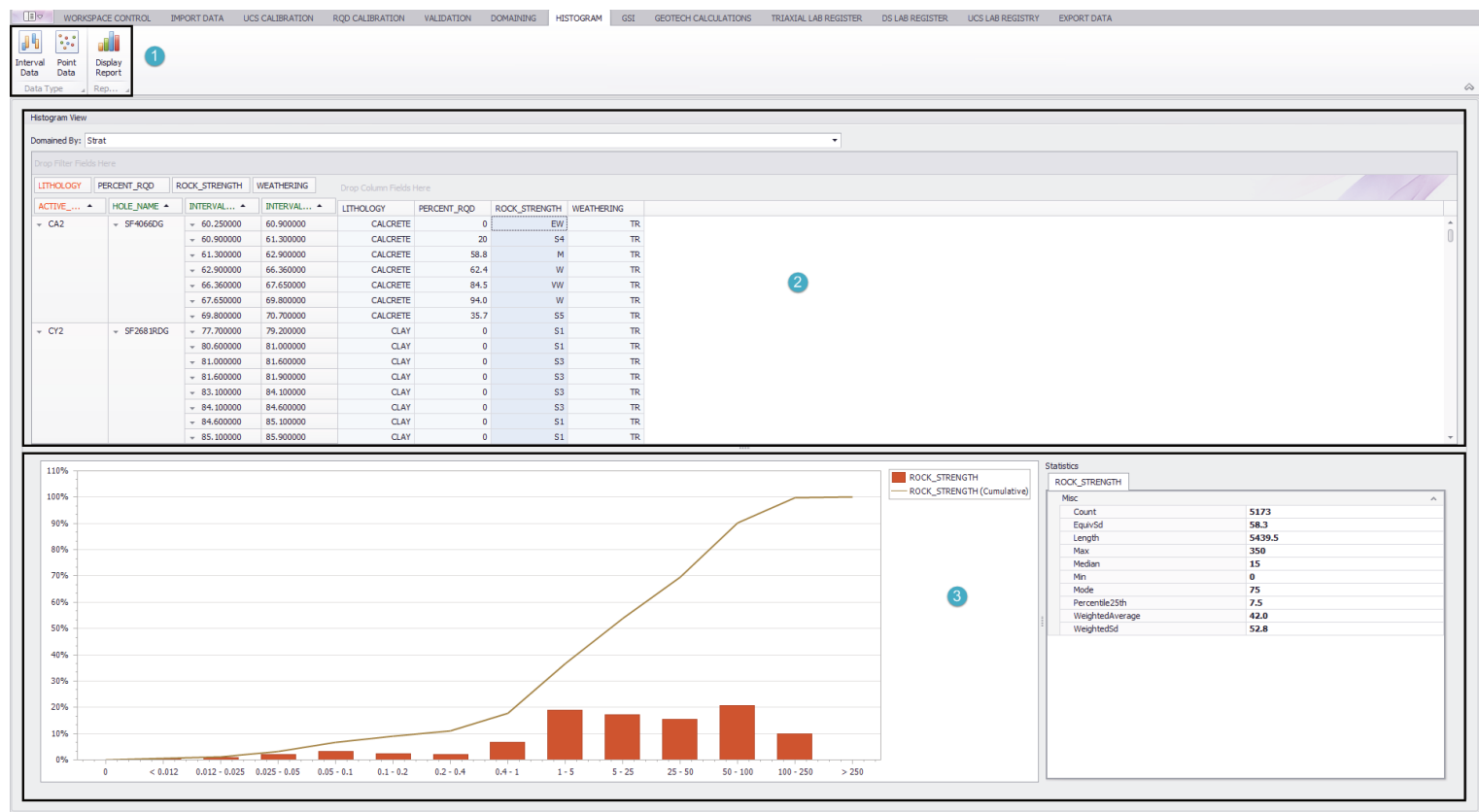

Figure 5 Rapid generation of histograms for logging data flagged using domain (or pre-domain) definitions based on stratigraphy, weathering, lithology or any other criteria. (1) The panel to select display of point or interval data, as well as reporting in PDF format; (2) A pivot table for actual dataset per hole and domain type; (3) The statistical analysis tool 


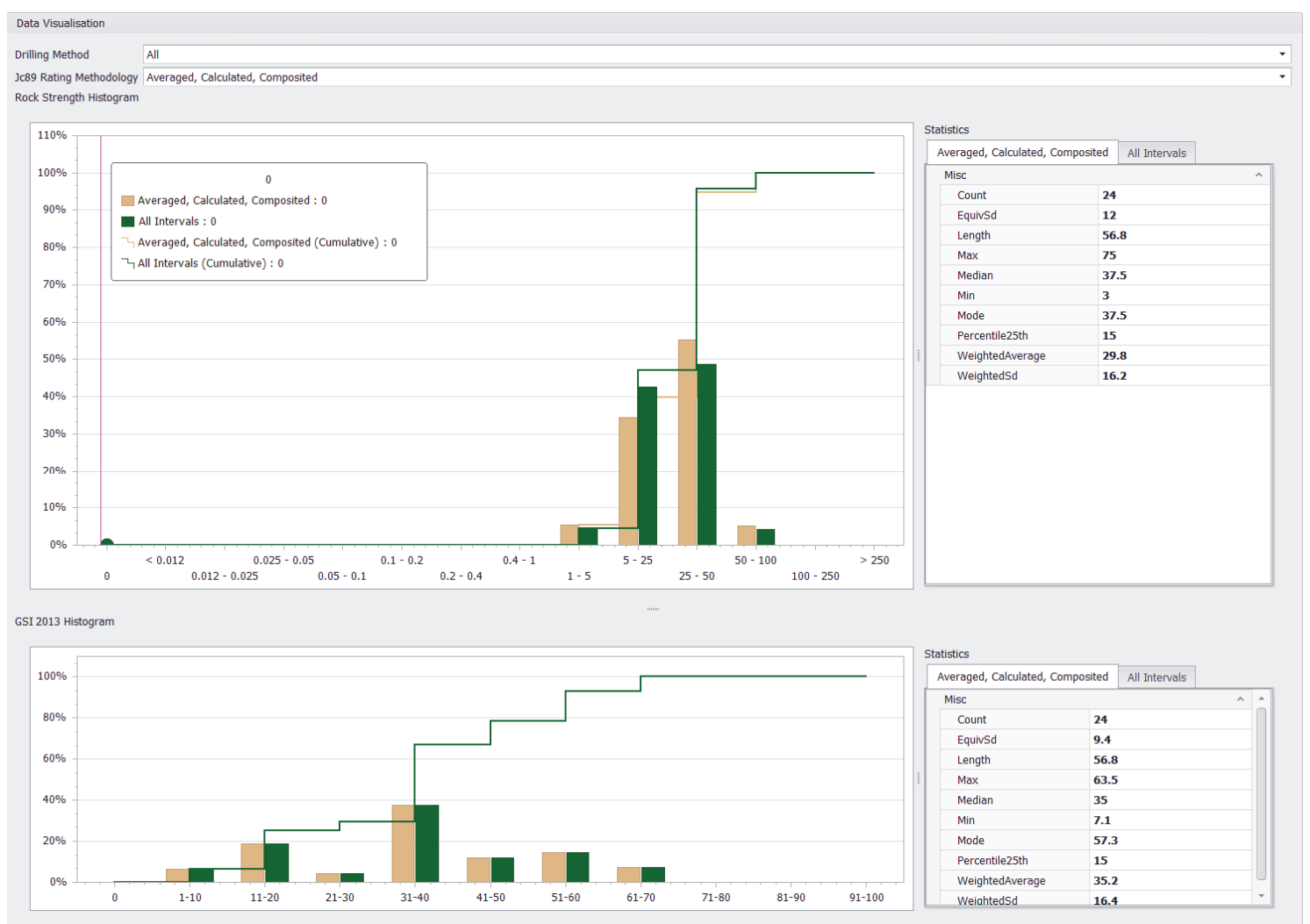

Figure 6 Calculation of geological strength index (GSI) and uniaxial compressive strength in parallel for same geotechnical rock mass domains. Statistics are presented next to histograms for rock strength (top histogram in MPa) and GSI (bottom histogram)

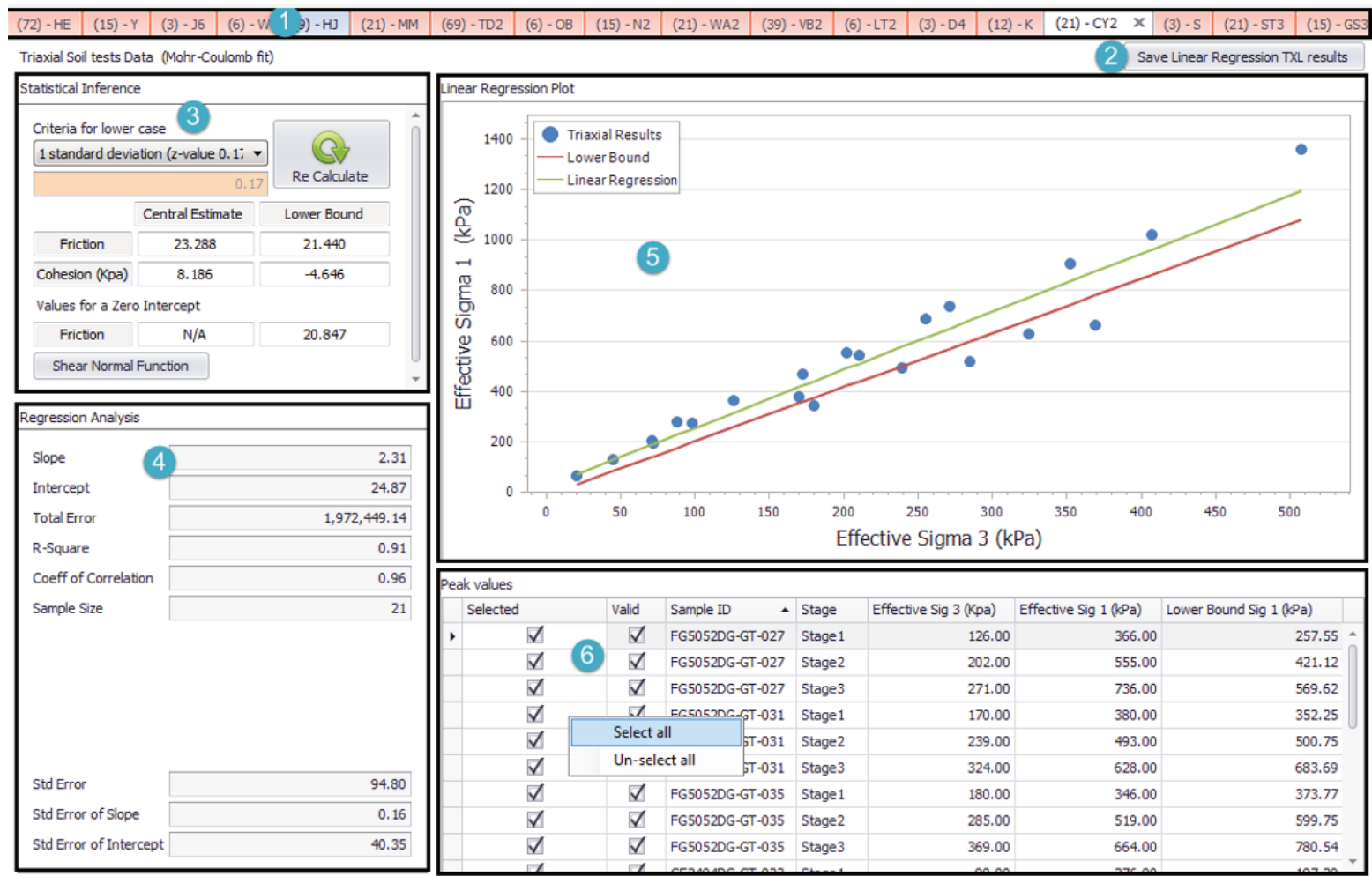

Figure 7 Calculation of linear regression best fit with prediction for lower bound based on percentiles approach. (1) Tab selector; (2) Button for saving data; (3) Strength estimates; (4) Regression analysis indicators; (5) Scatter plot (Mohr-Coulomb best fit); (6) The laboratory dataset 


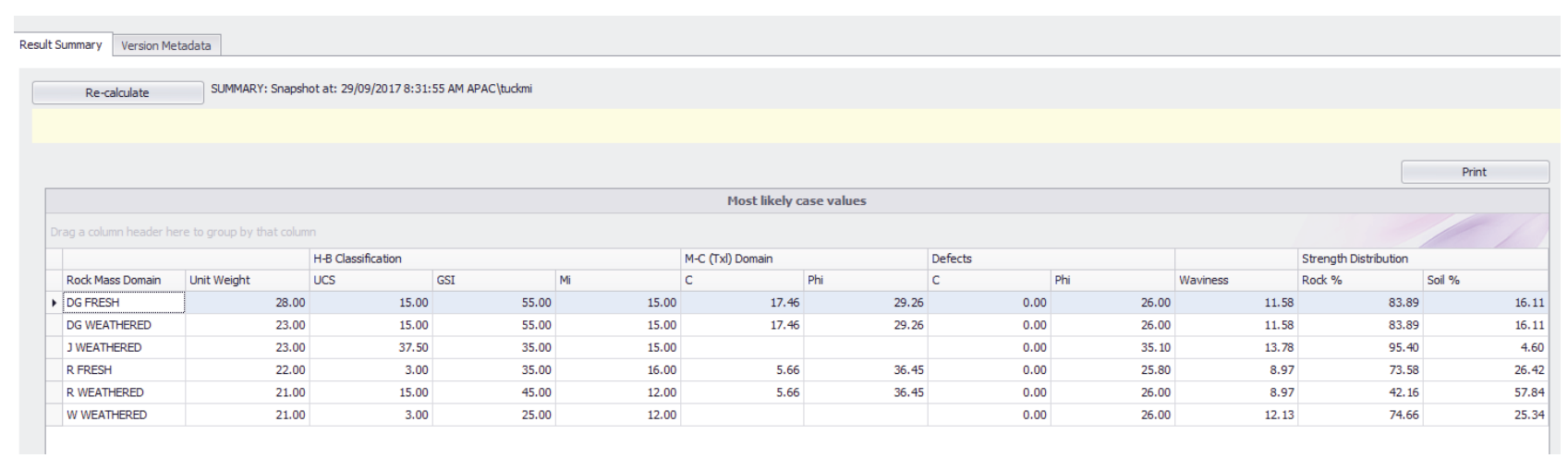

Figure 8 Summary table with all the values selected by users upon assessment of statistical analysis

\section{$4 \quad$ Benefits and possible replications}

Benefits include the following:

- Standard process that allows consistency in the design analysis.

- Time savings due to consistent and rapid process.

- One-stop for geotechnical data backup and server-based storage, eliminating the need for multiple excel spreadsheet files.

- The software works in the Windows ${ }^{\circledR}$ operating system. Its architecture is based on a collection of Ole objects that interact with visual net code. For copyright protection, the application only runs within BHP servers, however, the replication of Geotechnical Workbench is possible within other BHP commodities. Adaptation would require minor changes to the code whilst the essential architecture is likely to remain unchanged.

\section{Learnings}

The key learnings from software development include:

- The software was originally planned to be an aggregation of multiple modules. This permitted a progressive development of objects and a rational connection of temporal variables using in-memory calculations. Whilst this architecture permitted the development of individual modules in steady manner (e.g. less error), the adverse effect of this approach was the deferral of the final product.

- The development of the internal software was made possible by the following steps:

- Creation of an empty user interface (establish framework and setup of empty databases).

- Integration of empty platforms to existing external databases.

- Creation of individual modules within the empty interfaces.

- Testing of each module.

- This methodology proved to be efficient for coding.

- Development of software requires strong user engagement, especially for the development of user-friendly interfaces (e.g. not all users have coding experience).

- Simple answers do not necessarily mean a simple implementation. It is always possible to find non-compatible data. Commonly encountered errors include:

○ Stopping the system from rounding numbers on import.

- The existence of null values stopped computing many times. 
- Division by zero errors caused errors in the code and program stopped.

- These errors reduce data confidence and compromise the integrity of the software. Thus, it is very important to test the software with extreme situations and unusual data sets.

- Maintenance of open communication loops across the project team. Project management requires constant user feedback, repetitive testing and an ongoing search for simplicity.

- Communication loops don't have egos, whilst project leads should remain as being open-minded to accept changes in benefit of the project.

- Where possible, ongoing review to ensure original architecture still fits the purposes of the overall solution. However, in the experience of the author, it is always best to maintain a similar architecture after maturity.

\section{Acknowledgement}

The development of applications like Workbench are only possible due to the effort of multiple people including Tony Cockerill (project manager), Jeremy Abercrombie-Higgins (project manager), Andrew Haile (Sponsor), Chintana Meegamarachchi (senior developer), and Lukman Sasmita (senior developer).

The authors of this paper thank all the geotechnical engineers of BHP Iron Ore that have been constantly contributing with improvements to the software. Similarly, we thank the leadership team in resource engineering of BHP Iron Ore for supporting the development of Workbench for the geotechnical team, especially John Kirk for embedding software development capability, and Calvin Snodgrass for his continuous support throughout the development.

\section{References}

Barton, N, Lien, R \& Lunde, J 1974, 'Engineering classification of rock masses for the design of tunnel support', Journal of Rock Mechanics, vol. 6, no. 4, pp. 189-239.

Bieniawski, Z 1989, Engineering Rock Mass Classification, Wiley Interscience, New York.

Goodman, RE 1989, Introduction to Rock Mechanics, 2nd edn, John Wiley \& Sons, New York.

Hencher, S 2012, Practical Rock Mechanics, CRC Press, Boca Raton.

Hoek, E, Carter, TG \& Diederichs, MS 2013, 'Quantification of the Geological Strength Index Chart', Proceedings of the 47th US Rock Mechanics/Geomechanics Symposium, American Rock Mechanics Association, Alexandria.

Lancellota, R 2009, Geotechnical Engineering, 2nd edn, Spon Press, New York.

Maldonado, A 2013, 'Interpreting direct shear tests in the Pilbara environment - workshop in anisotropic materials', Workshop in Anisotropic Materials.

Maldonado, A \& Mercer K, 2015, 'An investigation into the shear strength of bedding planes in shale materials from the Hamersley Group rocks in the Pilbara region of Western Australia', Proceedings of the 2015 International Symposium on Slope Stability in Open Pit Mining and Civil Engineering, The Southern African Institute of Mining and Metallurgy, Johannesburg.

Marinos, P, Marinos, V \& Hoek, E 2007, 'Geological Strength Index (GSI). A characterization tool for assessing engineering properties for rock masses', in M Romana, A Perucho \& C Olalla (eds), Underground works under special conditions, Taylor \& Francis, Lisbon, pp. 13-21.

Snodgrass, C \& Cockerill, T 2017, 'Improving productivity integrating software developers into resource engineering', AUSIMM Bulletin, pp. 84-86. 